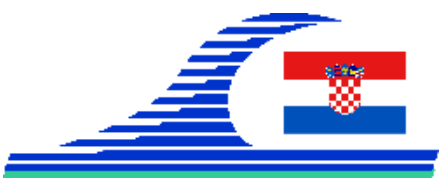

Conférence Méditerranéenne Côtière et Maritime

EDITION 4, SPLIT, CROATIA (2017)

Coastal and Maritime Mediterranean Conference

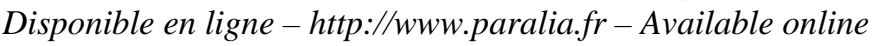

\title{
Mortier auto-plaçant à base d'un sédiment marin et de coproduits industriels
}

\author{
Philippe DHERVILLY ${ }^{1}$, Daniel LEVACHER ${ }^{2}$, Daniel RIVARD ${ }^{1}$, Hayat BOURTI ${ }^{1,2}$ \\ 1. Groupe Marais, 1 rue Pierre et Marie Curie, 49430 Durtal, France \\ p.dhervilly@samarais.com \\ 2. Université de Normandie, Unicaen, M2C UMR6143 CNRS, 24 rue des Tilleuls, \\ 14000 Caen, France. \\ daniel.levacher@unicaen.fr
}

\section{Résumé:}

L’installation de réseaux filaires dans le monde est en pleine expansion. Elle nécessite la réalisation de micro-tranchées peu profondes. Les sols excavés - déblais - ne sont jamais réutilisés pour leur remblaiement. Pour un développement durable, ces déblais constituent potentiellement une ressource en granulats pour le matériau de remblaiement qui pour ces travaux, doit être réexcavable i.e. posséder une résistance en compression minimale (2MPa) et maximale (4MPa). Devant la difficulté de le compacter, un matériau auto-plaçant convient. Un mortier auto-plaçant confectionné à partir de déblais de tranchées est étudié avec un liant à base de coproduits industriels pour éviter l'emploi de ciment afin de réduire l'empreinte carbone. Pour des travaux en zones portuaire et littorale, l'incorporation de sédiments marins issus de dragage a été envisagée. Un mortier à base de déblais de tranchées et de sédiments marins du port de Dunkerque a été élaboré pour en faire, après traitement, un matériau de remblai pour le comblement des tranchées, à la suite de la pose de réseaux filaires, sans besoin d'une mise en œuvre particulière. Du point de vue granulométrique, les matériaux excavés et les sédiments sont de nature limoneuse et sableuse. Des essais de caractérisation physique et mécanique du mortier à l'état frais et durci, ont été menés sur des échantillons prismatiques. L'affaissement au cône d’Abrams, l'ouvrabilité, la mesure des déformations de retrait et la résistance en compression simple. A l'état frais, la plasticité du matériau, doit être de classe S3 à S4, correspondant à un mortier auto-plaçant. La caractérisation, à l'état durci, du mortier auto-plaçant sans ajout de sédiments a montré une augmentation significative de la résistance en compression simple jusqu'à 28 jours. Les essais à l'état frais ont démontré une augmentation significative de l'ouvrabilité du mortier en ajoutant du sédiment marin dans les mélanges. A l'état durci, l'augmentation de la proportion de sédiments dans le mélange réduit le retrait dimensionnel à 28 jours mais diminue la résistance en compression.

Mots-clés : Mortier auto-plaçant, Déblais de tranchées, Sédiments marins, Coproduit industriel, Résistance en compression, Retrait. 
Mediterranean rocky coasts:

Features, processes, evolution and problems

\section{Introduction}

Actuellement, les sols excavés lors de la pose de réseaux ou canalisations sont mis en décharge et remplacés par des MAC (mortiers auto-plaçant) fabriqués dans des centrales à bétons à base de matériaux nobles issus de carrières. Une incitation au recyclage des déblais comme matériau de remblaiement est préconisée dans l'ensemble de l'Europe (EC, 2014). Elle permet de limiter dans le temps l'installation de plateformes ou de centres de stockage de déblais mais aussi l'économie de ressources en granulats en les remplaçant par ces déblais excavés qui devront être traités pour atteindre les performances mécaniques nécessaires à leur réemploi. Dans le cas du comblement de tranchées, il s'agit de confectionner un matériau fluide i.e. auto-plaçant (BOUHAMOU et al., 2014 ; OUCHI et al., 1997 ; OUCHI, 2001) pour ne pas être compacté dont la résistance à la compression doit être au moins égale à $2 \mathrm{MPa}$ tout en étant par la suite réexcavable (AFNOR, 2009). Pour un développement durable et responsable, un mortier auto-plaçant composé de déblais de tranchée a été étudié en combinant ces déblais à des sédiments marins et des liants issus de coproduits industriels i.e. des laitiers. La problématique des sédiments dragués et gérés à terre s’apparente à celle des déchets comme celle des déblais. En zone littorale à forte densité de population et portuaire industrielle où les réseaux sont nombreux, ces mortiers de comblement additionnés de sédiments marins peuvent constituer une voie de valorisation pour des petits volumes. L'étude du mortier présentée concerne un mortier composé de déblais de tranchée, de laitiers et d'un sédiment marin issu du Port de Dunkerque. L'analyse porte sur les performances physico-mécaniques obtenues.

\section{Matériaux et méthodologie}

La formulation du mortier de référence i.e. témoin MAP T, contient $86.5 \%$ de déblais, 16\% d'eau, $12 \%$ d'ACTi200 (mélange de laitiers) et $1.5 \%$ de chaux avec un rapport eau/liant E/L de 1.185. Les mélanges (déblais + sédiment + liant + eau), constituent les mortiers MAP i\% avec i\% comme ajout de sédiments. La teneur en eau initiale des sédiments était de $130 \%$, du point de vue pratique aucun séchage des sédiments n'est prévu. Ils ont donc été incorporés à cette teneur dans les mélanges. Les sédiments SDK (Dunkerque, France) et les déblais de Bû (Eure-et-Loir, France), correspondent à un matériau argilo-limoneux fin, voir tableau 1, (AFNOR, 1999).

Tableau 1. Quelques caractéristiques des mortiers, déblais et sédiments.

\begin{tabular}{|l|l|l|l|l|l|l|l|}
\hline $\begin{array}{l}\text { Référence } \\
\text { mortier }\end{array}$ & $\begin{array}{l}\text { Eau/Liant } \\
\text { E/L }\end{array}$ & $\begin{array}{l}\text { Affaissement } \\
(\mathrm{cm})\end{array}$ & $\begin{array}{l}\text { Consistance } \\
\text { Classe Si }\end{array}$ & $\begin{array}{l}\text { Fraction } \\
\text { minérale }\end{array}$ & $\begin{array}{l}\text { Diamètre } \\
(\mu \mathrm{m})\end{array}$ & $\begin{array}{l}\text { Déblais } \\
\text { Bû }(\%)\end{array}$ & $\begin{array}{l}\text { SDK } \\
(\%)\end{array}$ \\
\hline MAP T & 1.18 & 12.00 & Plastique S3 & Argiles & $<2 \mu \mathrm{m}$ & 11 & 14 \\
\hline MAP 5 \% & 1.55 & 14.25 & Plastique S3 & Limons fins & $2 \mu \mathrm{m}-20 \mu \mathrm{m}$ & 39 & 40 \\
\hline MAP 10\% & 1.91 & 17.50 & Fluide S4 & Limons grossiers & $20 \mu \mathrm{m}-50 \mu \mathrm{m}$ & 30 & 14 \\
\hline MAP 20\% & 2.64 & 20.00 & Fluide S4 & Sables fins & $50 \mu \mathrm{m}-200 \mu \mathrm{m}$ & 14 & 21 \\
\hline MAP 30\% & 3.37 & 23.75 & Très fluide S5 & Sables grossiers & $200 \mu \mathrm{m}-2 \mathrm{~mm}$ & 6 & 11 \\
\hline
\end{tabular}


Mediterranean rocky coasts:

Features, processes, evolution and problems

Des essais sur éprouvettes prismatiques $4 \times 4 \times 16 \mathrm{~mm}^{3}$ ont été menés afin d'étudier la résistance en compression après une période de cure à température ambiante $\left(20 \pm 2^{\circ} \mathrm{C}\right)$ de 7, 14 et 28 jours, (AFNOR, 2016 ; AFNOR, 2012).

\section{Performances physico-mécaniques des mortiers}

\subsection{Résistance à la compression simple}

Après avoir soumis les éprouvettes prismatiques à l'essai de traction par flexion, l'essai de compression est effectué sur des cubes $4 \times 4 \mathrm{~cm}^{2}$, au nombre de 6 par mélange. Tout d'abord les mortiers suivent la même cinétique de durcissement en fonction du temps entre 7 et 28 jours de cure (figure 1, en haut), on constate que l'incorporation de sédiments affecte la résistance. On observe sur la figure 1 (en bas) que le dosage en sédiments fait chuter la résistance de manière linéaire, quelle que soit l'étape de durcissement jusqu’à 28 jours.
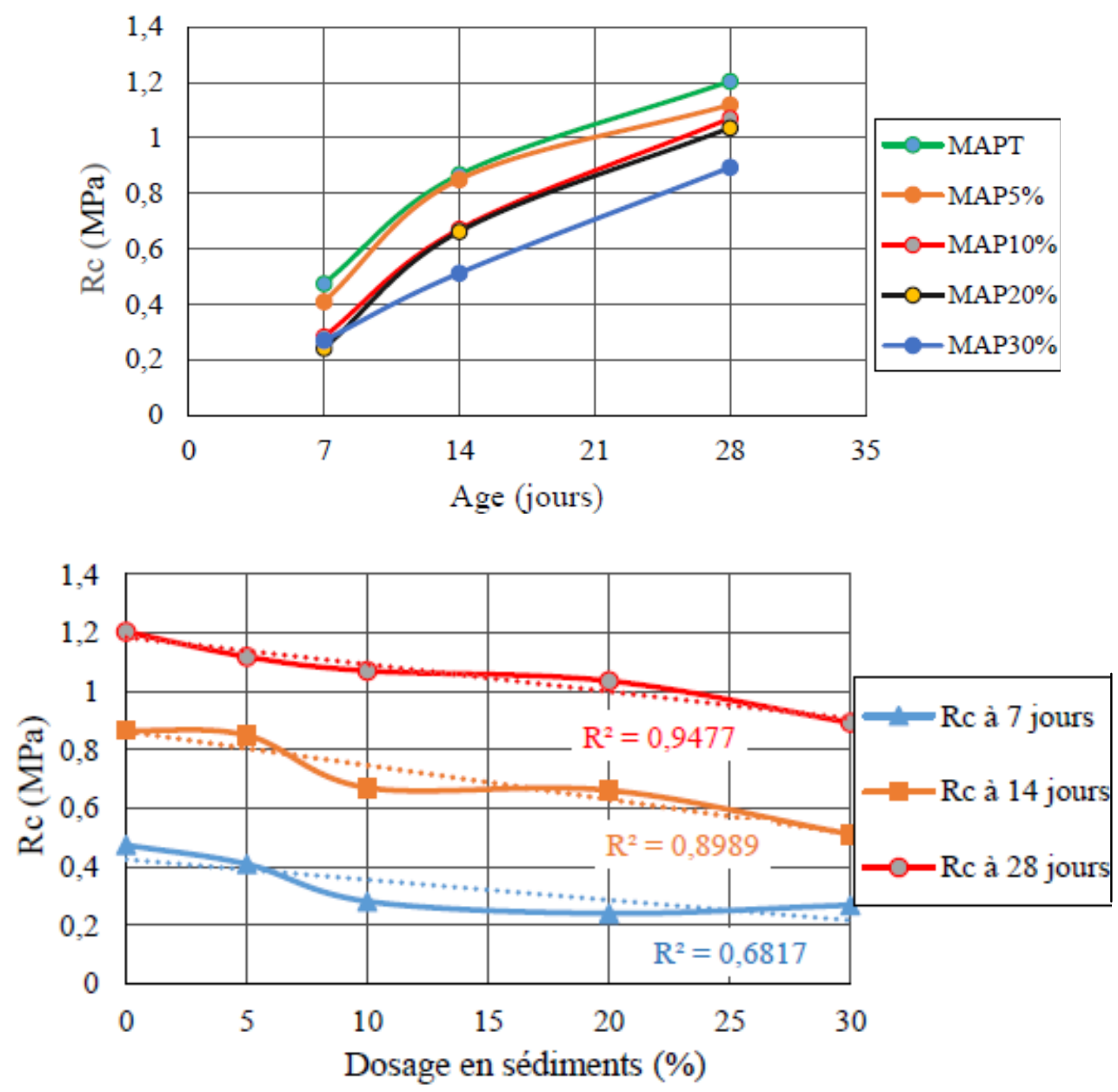

Figure 1. Résistance à la compression des mortiers

En haut, durcissement en fonction du temps ; en bas, effet du dosage en sédiments. 
Mediterranean rocky coasts:

Features, processes, evolution and problems

Le mortier témoin MAPT développe la meilleure résistance mais en deçà de la valeur de 2MPa requise. Cependant la cinétique de durcissement montre qu'à 28 jours, les résistances n'ont pas atteint la valeur maximale, ceci est en mettre en relation avec la pouzzolanicité du liant utilisé (combinaison de laitiers). La consistance initiale des mortiers i.e. le rapport $\mathrm{E} / \mathrm{L}$ est un paramètre qui reste à optimiser. Toute augmentation en eau conduit aussi à une diminution de la résistance, figure 2 à gauche.

Si l'on observe l'évolution des résistances à la compression à 28 jours, (figure 1, en bas), l'incorporation de sédiments à des taux faibles jusqu'à 10\% n'entraîne qu'une réduction de $10 \%$ de la résistance.
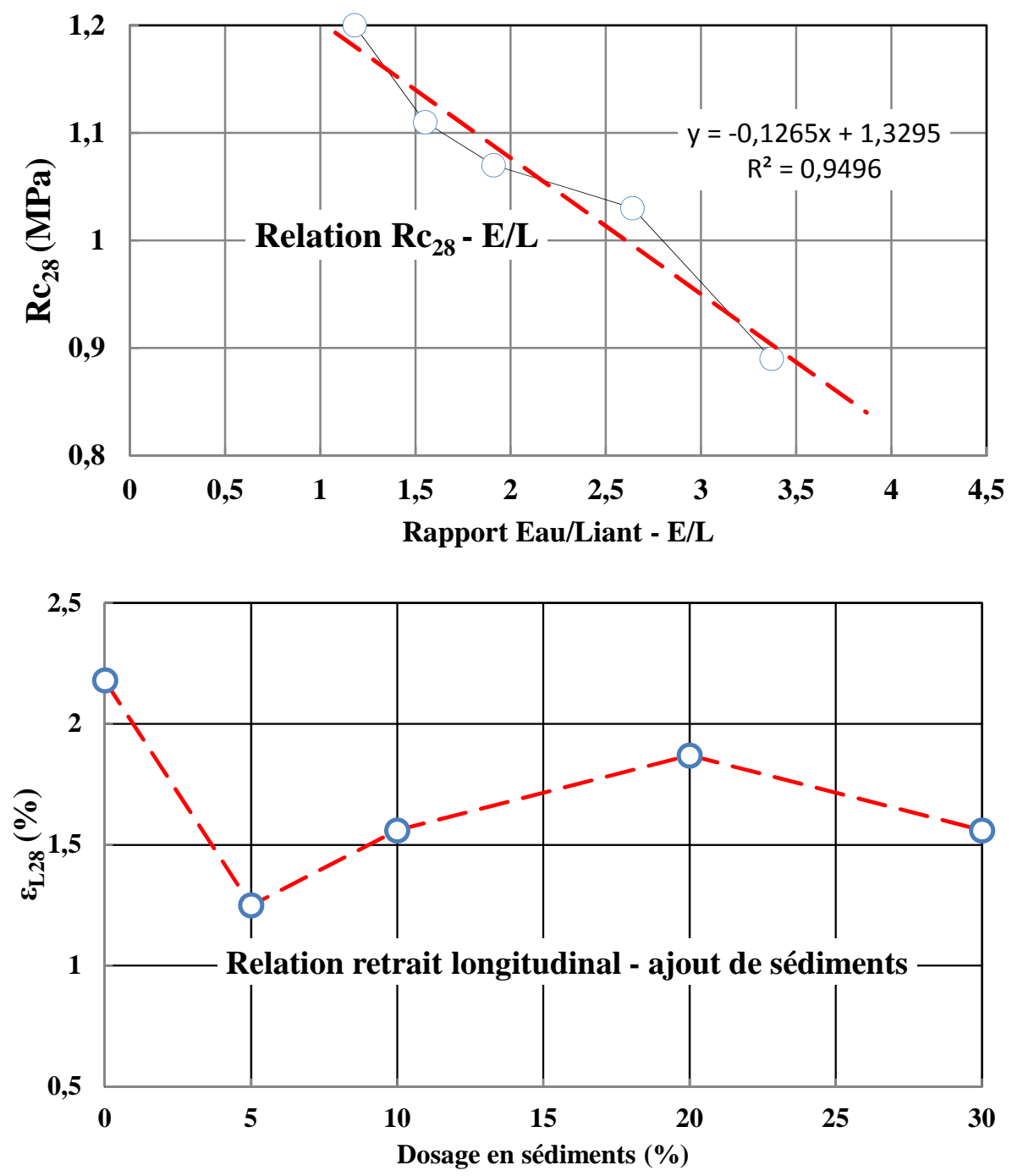

Figure 2. Caractérisation des mortiers

En haut, influence du rapport eau/liant ; en bas, retraits observés à 28 jours. 
Mediterranean rocky coasts:

Features, processes, evolution and problems

\subsection{Variations dimensionnelles}

Lors de la mise en place d'un mortier auto-plaçant en remplissage de tranchées, le contrôle porte sur son ouvrabilité (consistance) et sa résistance en compression mais aussi sur la connaissance du retrait du matériau une fois mis en place.

Les mesures systématiques des dimensions des éprouvettes prismatiques effectuées avant les essais mécaniques ont permis d'évaluer les variations dimensionnelles et volumiques durant la période de cure et d'apprécier globalement le phénomène de retrait. Du point de vue phénoménologique, on note une évolution rapide du retrait aux jeunes âges (phases d'hydratation et séchage) jusqu'à 7 jours puis un net ralentissement par la suite pour tous les mélanges. Les variations dimensionnelles observées sur les mortiers testés par rapport au mortier témoin MAPT, sont étroitement liées à l'augmentation de la proportion de sédiments incorporés et d'autre part, à l'accroissement du rapport $\mathrm{E} / \mathrm{L}$. Mais le sédiment peut jour le rôle d'un filler : améliorer l'ouvrabilité et réduire le retrait. On observe à 28 jours sur la figure 2 de droite que l'apport de sédiments tend à réduire le retrait longitudinal (sur éprouvette prismatique) mais le stabiliserait même si le rapport E/L croît en fonction du dosage en sédiments.

\section{Conclusions}

Cette étude sur mortiers à base de liants issus de coproduits industriels (laitiers) montre qu'il est potentiellement possible de recycler des déblais de tranchées avec éventuellement ajout de sédiments. Elle montre aussi toute la difficulté de trouver une formulation adéquate pour obtenir des performances suffisantes aussi bien en termes de consistance, de retrait et de résistance en compression.

Un mélange qui incorpore une quantité de $1.5 \%$ de chaux (action sur les particules fines argilo-limoneuses) et $12 \%$ de liant combinant des laitiers peut constituer une formulation de base. Elle peut être ajustée, voir complétée à l'aide d'activateurs pour atteindre une résistance de $2 \mathrm{MPa}$ très tôt vers 7 jours. L'addition de sédiments dragués dans ces mortiers peut être opérée lors de remblaiement en zone littorale ou portuaire. Les sédiments peuvent influer sur l’ouvrabilité (plasticité et consistance, rôle de filler) et par de même sur la compacité du mortier et le retrait comme observé.

\section{Références bibliographiques}

AFNOR - NF EN 12350-2. (2012). Essai pour béton frais - Partie 2 : Essai d'affaissement. Avril 2012, P 18-431-2.

AFNOR - NF EN 196-1. (2016). Méthodes d'essais des ciments - Partie 1 : détermination des résistances - Méthodes d'essais des ciments - Partie 1 : Détermination des résistances. Septembre 2016, P15-471-1.

AFNOR - XP P94-011. (1999). Sols : reconnaissance et essais - Description. Identification. Dénomination des sols - Terminologie. Éléments de classification. Août 1999, P94-011. 
Mediterranean rocky coasts:

Features, processes, evolution and problems

AFNOR - XP P98-333. (2009). Chaussées et dépendances : Tranchées de faibles dimensions, micro tranchées. Norme expérimentale. Juin 2009, P98-333.

BOUHAMOU N.-E., MOSTEFA F., MEBROUKI A., BELARIBI N., MESBAH A.H. (2014). Etude du comportement d'un mortier autoplaçant à base des sédiments de dragage vis- à-vis du retrait. MATERIAUX 2014, Colloque Ecomatériau, Nov. 2014, Montpellier, France. hal-01144447

BOURTI H. (2017). Etude d'un mortier auto-plaçant à partir de déblais de tranchée et de sédiments marins, Rapport Master 2 IGL, Université de Normandie-Unicaen, 62p.

EC- EUROPEAN COMMISSION (2014). Towards a circular economy: A zero waste programme for Europe, DG Environment, Minsk, 8 October 2014, www.oecd.org/env/outreach/EC-Circular-econonomy.pdf

OUCHI M. (2001). Self-compacting concrete: development, applications and key technologies. $26^{\text {th }}$ Conference on Our World in Concrete \& Structures, 27 - 28 August 2001, Singapore, Article Online Id: 100026008, 2001, pp. 89-97. http://cipremier.com/100026008

OUCHI M., HIBINO M., OKAMURA H., (1997). Effect of superplasticizer on selfcompactibility of fresh concrete. Journal of the Transportation Research Board, Vol. 1574, pp. 37-40, doi: 10.3141/1574-05

RECYSOILS $^{\circledR}$. (2017). Projet AMI ADEME Recyclage - FEDER Régional, Groupe MARAIS, 49430 Durtal, France). Document confidentiel. http://www.samarais.com/fr/ 